\title{
Chirping Fibre Bragg gratings within additively manufactured polymer packages
}

\author{
JOHN CANNING, ${ }^{1,}{ }^{*}$ ASMA ZIYANI ${ }^{1,2}$ \\ ${ }_{1}^{1}$ interdisciplinary Photonics Laboratories (iPL), School of Electrical \& Data Engineering, Tech Lab, University of Technology Sydney, NSW 2007 \& \\ 2019, Australia \\ ${ }^{2}$ Polytech Paris-Sud, Université Paris-Sud, Universite Saclay, Orsay, Paris, France \\ *Corresponding author: John.Canning@uts.edu.au
}

Received XX Month XXXX; revised XX Month, XXXX; accepted XX Month XXXX; posted XX Month XXXX (Doc. ID XXXXX); published XX Month XXXX

Fibre Bragg gratings are embedded within 3D printed polymer packages. Information about both induced and applied stresses, and operator error, can be determined from the observed spectral shifts and chirping. A novel way to produced packaged broadband gratings, with $\Delta \lambda>$ 7nm/cm, is proposed and demonstrated. (C) 2019 Optical Society of America

http://dx.doi.org/10.1364/OL.99.099999

The combination of additive manufacturing and fibre Bragg gratings (FBG) potentially addresses the high cost involved with labor intensive FBG and optical fibre sensor packaging. By embedding sensor elements such as FBGs within 3D printed materials, a sensor can be customised for different applications with unprecedented novelty and ease. For example, 3D printed soft, flexible plastic insoles embedded with FBGs enable smart sensing for animal and human gait diagnostics [1-3]. 3D printing using low cost methods such as fused deposition modelling (FDM) can enable stiff, tough packages suitable for harsh environments. FDM created packages are being used to protect fibre gratings both mechanically and from water or chemical permeation across a range of infrastructure from buildings to railway tracks. In most of these examples, the optical fibres are fixed, without additional effort, because polymers have higher density solid states compared to their liquid states, leading to compression on the fibre. This greatly relaxes complexity and, with the appropriate material design possible using additive manufacturing, can negate the need for pre- or posttreatment methods. Such potential contrasts greatly with commercial work on developing FBG packaging [4,5], culminating in, for example, metal-based CNC machined packages of considerable sophistication exploiting tensile loads needed to obtain zero temperature and strain sensitivity. Such packages are inherently costly making optical sensors broadly prohibitive. In this paper, we focus on exploring FBG packaging within available materials used in low cost additive manufacturing, in part to address the fact that cost remains a deciding factor in the adoption of optical waveguide-based sensing.

Generally, there has been little research in establishing the preparation conditions of these 3D printed packages despite the sensitivity of polymer and other material systems to processing and preparation conditions. Notably, the densification of the solid state depends heavily on various relaxation rates including the temperature, energy and time involved. To investigate this and reproducibility, we focus on FDM printing and examine multiple optical fibre Bragg grating (FBG) samples embedded within 3D printed acetylene butadiene styrene (ABS). A reference FBG sample where minimal if any tension is applied to the fibre Bragg grating is placed above an initial layer of printed ABS film. $45^{\circ}$ $x, y$ printing back and forth is used to build up the layer in the $z$ direction, known to enable strong packages. A second overlay over the sample is then created, effectively trapping and fixing the FBG sample in place with a soft jaw-like configuration that can substantially enhance the compressive grip of the solid state.

A net consequence is a slight compression on the FBG is observed for a straight but unstrained sample arising from the denser state of the polymer after solidification. Provided there is no applied strain this should be observed as a simple shift in grating peak with little change in grating profile, providing material density information. In this paper, we also quantify the variance in error of this by monitoring chirp and working backwards estimating the actual applied stretching force on the FBG and optical fibre. The work is undertaken by the student (Author 2) to be consistent in source variance and under controlled conditions the changes in spectral shape are used to determine the presence of load. This spectral shape is directly translated as an applied chirp arising for an existing force gradient (the applied tension deviating from zero) - working from the spectra, the applied force can be quantified. The variance in this is used to then quantify an operator error and the limits of a human performed function. We use this approach to justify the call for automation and the replacement of human labour in next generation photonic technologies. 


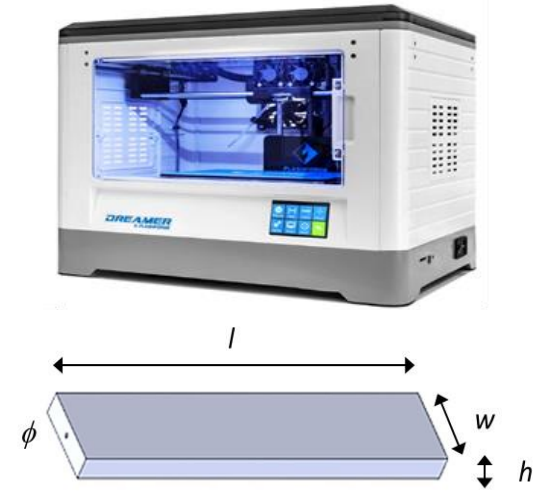

Fig. 1. A low-cost budget fused deposition modeling (FDM) 3D printer was used in the work reported here. A schematic of a printed package dimensions is illustrated below.

Figure 1 shows the printer (Flashforge Dreamer) used in this work, selected because it is low cost, reliable and used successfully for photonics including printing the first optical preforms for plastic fibre $[6,7]$.

All non-damage gratings $\left(\lambda_{\mathrm{B}} \sim 1546.3 \mathrm{~nm}\right)$ were standard readily supplied $10 \mathrm{~mm}$ long devices (direct writing: $\operatorname{ArF} \lambda=$ $193 \mathrm{~nm}$ exciplex emission, standard conditions: pulse fluence $f_{\text {pulse }} \sim 70 \mathrm{~mJ} / \mathrm{cm}^{2}$, cumulative fluence $f_{\text {cum }} \sim 85 \mathrm{~J} / \mathrm{cm}^{2}$ ) into germanosilicate (Nufern-like GF1 [GeO $]$ 12 mol\%) [8-12]. Figure 2 (a) shows an example of a typical grating spectrum. Measured data is collected using a commercial interrogator and extracted as images post-analysed for this work ( $\Delta \lambda$ res $=0.001 \mathrm{~nm} ; \Delta \lambda$ variability $\sim 0.01 \mathrm{~nm}$ ). These gratings are then introduced within the 3D printing process so that they are embedded within layers through compressive force upon solidification of the printing polymer. Different applied tensions should lead to different compressive effects including chirping of the grating given the FDM printer scans from one side to the other in the zig-zag writing configuration.

Standard fused deposition modelling (FDM) based additive manufacturing is undertaken using a common thermoplastic (acrylonitrile butadiene styrene - ABS; solid density varies because of varying proportions of constituents over $\rho \sim(0.9$ - 1.53) g/ $\mathrm{cm}^{3}$ with a median $\sim\left(1.060\right.$ - 1.080) g.cm ${ }^{-3}$ $[13,14])$. A package was printed layer by layer. The FBG was placed on one layer before a secondary layer was printed above. The FDM passes roughly at $45^{\circ}$ over the fibre axis. An image of the completed package (length $L=60 \mathrm{~mm}$, thickness $\sim 2 \mathrm{~mm}$ ) is illustrated in Figure 2 (c). Since the density of the solid state is higher than the liquid state the FBG is under compression in the final packaged. Figure 2 (b) shows the shift to shorter wavelength observed over the compression of the FBG over $1 \mathrm{~cm}-\Delta \lambda_{\mathrm{B}} \sim 0.156 \mathrm{~nm}$. There is a slight spectral broadening but otherwise the grating remains largely uniform.

Given this uniformity, one can estimate from the shift to shorter wavelengths a percentage increase in density of the package of approximately $1 \%$ (or $\sim 0.01 \mathrm{~g} / \mathrm{cm}^{3}$ from the median) when solidified. The practical ramification of this density increase is a compressive fixing of the FBG within the package, negating the need for epoxies or other materials.
Another consequence is the appearance of chirp in the grating spectrum if there is an applied tension on the fibre during printing. Such chirping can also be deliberately introduced - for example, applied stress during the directional placement of epoxy along a grating has previously been exploited to create chirped gratings [15], to broaden the spectral filter or to enable dispersion compensation or temporal dilation.
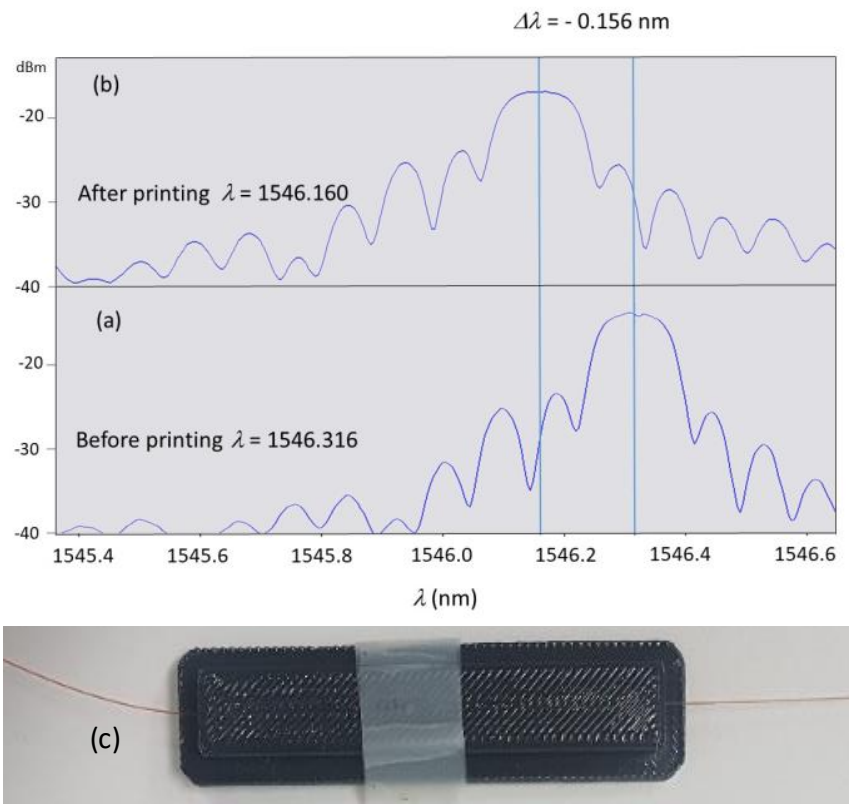

Fig. 2. Uniform fibre Bragg grating spectra written by direct writing with $193 \mathrm{~nm}$ before (a) and after (b) packaging. The final package is illustrated in (c) where the $45^{\circ}$ passes are visible (Tape visible in centre is a label). The slight uniform broadening can be attributed in part to the

We explored the potential of unintentionally locking in, through polymer compression, applied strain applied to an FBG when stretching along the fibre for inducing chirp into the grating spectra. By examining arbitrary chirping induced during preparation, it is possible to forensically extract the applied tension and operator variability as well as sensitivity. Variability as a measure of reproducibility of the packaging process can provide a quantifiable assessment of human operator limitations. For example, in Figure 3 several chirped samples of varying chirp were readily, but unintentionally, fabricated by the operator when the applied tension is not systematically considered. The lack of control of this tension led to variable spectral chirping over a significant range between $\Delta \lambda /=0$ and $7 \mathrm{~nm} / \mathrm{cm}$. This corresponds to a delay of $\tau=0.7 \mathrm{ps}$ for reflected light from the front to the back, something that can be exploited as a delay line device or for dispersion compensation. The integrated area under the graphs is nearly constant, reflecting a drop in peak as the spectrum is distributed over a wider spectrum. This is a good indication that only the applied tension is responsible for the chirping and there is no major induced loss or fibre damage occurring. The direction of the spectral tilt depends also on the direction of printing relative to the applied stress. The spectral shift towards shorter wavelengths is consistent with net variation in compression along the FBG. From an operational perspective, removing human variability should 
dramatically improve consistency and uniformity of results by removing any tension beyond that required to keep the fibre straight.

The origin of chirp arises from the direction of printing relative to the applied tension (done manually by hand). When writing is in the direction of the applied load, the grating chirp tends to lead to stronger peaks and to shorter wavelength whereas when tension was applied away from the writing, the grating after initial printing experiences a less varied applied load. These results are consistent with past results based on manual deposition of epoxy along the grating by Hill [15]. Both the mechanism and human source of error can be accommodated through automation where robots apply constant tension and 3D printing provides constant packaged deposition. Successful deployment in robotic packaging of photonic products is not new having been demonstrated by Kadence Photonics for the production of fibre thermal beam expanders, demonstrating one of the earliest industrial IoT applications in photonics and introducing the concept of industrial "fembots" [16].
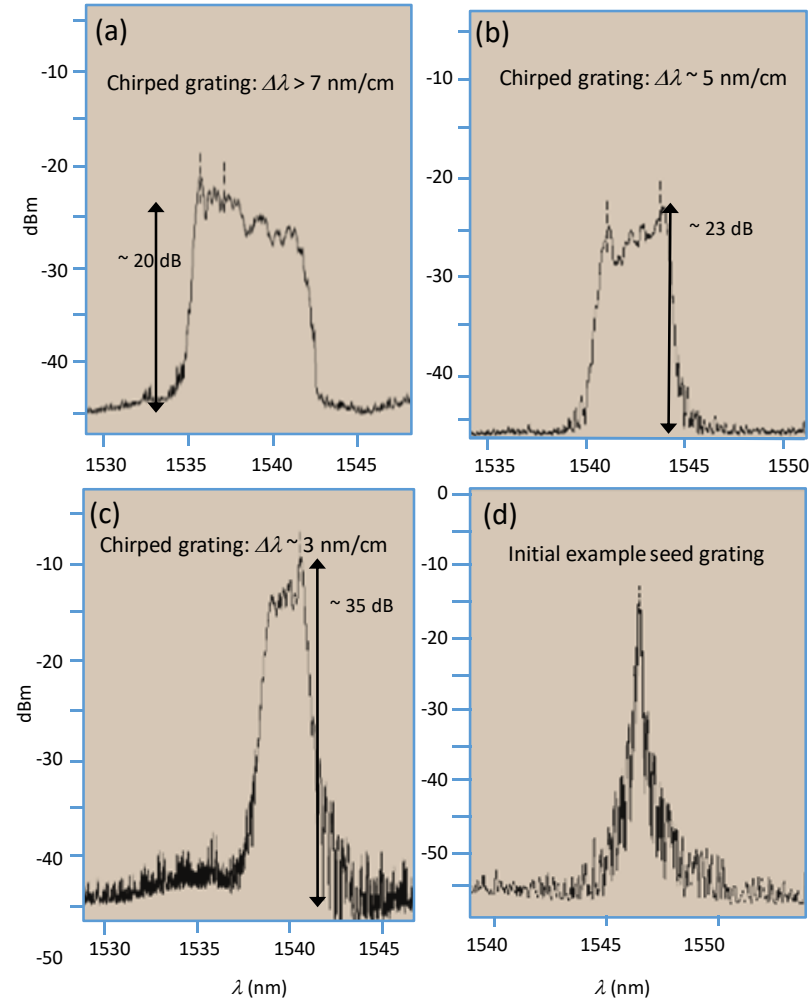

Fig. 3. Applying load stretches the FBG. Under compression packaging that load translates into a chirped spectrum as the applied tension varies during printing over one end of the FBG to the other. From (a) to (c) the spectrum bandwidth can be tuned beyond $\Delta \lambda L=7 \mathrm{~nm} / \mathrm{cm}$. (d) Shows the uniform grating spectra measured with no chirp and low interrogator resolution.

Table 1 summarises a series of results obtained with fabrication of the packaged gratings as described above without any systematic control or recording of applied tensions to assess variability of the human operator. A number of parameters were varied to see if any additional factors are involved, including packaging length, temperature and cooling rate. None of these impacted on their own results in any way that could not be predicted. This along with the similar integrated areas under the spectra, pointed towards human operator variability as the major source of variance in the results. Given the need for low labour costs, this suggests the need for full automation of FBG fabrication in practice.

Alternatively, there is a clear opportunity to introduce arbitrary chirp profiles deliberately for applications as diverse as tailored bandwidth filter widths, delay taps and dispersion compensation [17]. Further, with appropriate design and physical bending, 3D printing readily enables tunable components analogous to that achieved with greater difficulty by traditional means [18].

In conclusion, we have shown an assessment of 3D printed packaging. In the first instance, without good control of human operator, the semi-automated packaging involving 3D printing leads to a great deal of variability or error. This source of error arises from applied tension on the fibre that manifests itself in spectral broadening and short wavelength shift of the grating. It arises from variable compression along the FBG. Because the source of error is well defined, it is possible to correct and remove quite readily in specialised facilities where, for instance, humans are replaced with robots to complement 3D printing's automative integrateability (an anticipated prerequisite for industrial IoT). For manufacturing packaged uniform gratings, further automation is therefore required. This work also opens up novel opportunities to further advance grating design and packaging. It can be exploited, for example, as a novel tool for creating chirped gratings where high rates of chirp were achieved with little effort $(\Delta \lambda \sim 7 \mathrm{~nm} / \mathrm{cm})$. With planned tension, the potential values can be much higher still. These chirped devices can act, for example, as broadband filters, dispersion compensators or tunable variable delay lines.

Acknowledgements. A. Ziyani acknowledges supply of direct written gratings and assistance with 3D printing from Kevin Cook.

Funding. This work was supported by private funds. A. Ziyani acknowledges financial support from Universite de Paris Sud.

Disclosures. The authors declare no conflicts of interest.

\section{References}

1. D. Vilarinho, A. THoodoiou, C. Leitao and C. A. F. Marques, Sensors 7 (12), 2924, (2017).

2. Z. Hao, K. Cook and J. Canning, $8^{\text {th }}$ Asia Pacific Opt. Sensors Conf. (APOS 2019), Auckland, New Zealand (2019).

3. C. Martelli, J. Carlos Cardozo da Silva, D. Pipa, M. José da Silva, C.R. Zamarreño, J. Canning, P. E. Schaphauser, G. Dutra, R. Daciuk, J. R. Galvão, F. Mezzadri, A. B. Renzo, U. Dreyer, G. Weber, C. Janeczko and O. G. da Rocha, SPIE Proc. V10323, 25th Int. Conf. on Optical Fiber Sensors, 103230W (2017). https://doi.org/10.1117/12.2272465

4. G. W. Yoffe, Peter A. Krug, F. Ouellette, and D. A. Thorncraft, Appl. Opt. 34, 6859-6861 (1995).

5. D.C. Psaila, H.G. Inglis, IEEE Proc. $51^{\text {st }}$ Electron. Components \& Tech. Conf. (2001).

6. K. Cook, G. Balle, J. Canning, L. Chartier, T. Athanze, M.A. Hossain, C. Han, JE. Comatti, Y. Luo and G-D. Peng, Opt. Lett., 41 (19), 4554-4557, (2016). 


\begin{tabular}{|c|c|c|c|c|c|c|c|c|c|}
\hline \multicolumn{10}{|c|}{ Package Length } \\
\hline $\begin{array}{c}L \\
(\mathrm{~mm})\end{array}$ & \multicolumn{3}{|c|}{40} & \multicolumn{3}{|c|}{60} & \multicolumn{3}{|c|}{80} \\
\hline $\begin{array}{c}\lambda \\
(\mathrm{nm}) \\
\text { before printing }\end{array}$ & 1546.358 & 1546.545 & 1546.52 & 1546.3 & 1546.5 & 1546.5 & 1546.4 & 1546.3 & 1546.5 \\
\hline $\begin{array}{c}\qquad \\
\qquad(\mathrm{nm}) \\
\text { after printing }\end{array}$ & 1537.538 & 1546.464 & 1546.369 & 1546.2 & 1546.2 & 1546.5 & 1546.3 & 1545.5 & 1546.4 \\
\hline $\begin{array}{c}\Delta \lambda \\
(\mathrm{nm})\end{array}$ & 8.82 & 0.081 & 0.151 & 0.122 & 0.29 & 0.021 & 0.02 & 0.781 & 0.013 \\
\hline$\Delta \lambda / \lambda=\Delta L / L$ & 0.005703724 & $5.23748 \mathrm{E}-05$ & $9.76386 \mathrm{E}-05$ & $8 \mathrm{E}-05$ & 0.0002 & $1 E-05$ & $1 \mathrm{E}-05$ & 0.0005 & $8 E-06$ \\
\hline $\begin{array}{l}\Delta \mathrm{\lambda}_{o v} \\
(\mathrm{~nm})\end{array}$ & \multicolumn{3}{|c|}{$3.0 E+00$} & \multicolumn{3}{|c|}{$1.4 \mathrm{E}-01$} & \multicolumn{3}{|c|}{ 2.7E-01 } \\
\hline$(\Delta \lambda / \lambda)_{o v}$ & \multicolumn{3}{|c|}{$1.95 \mathrm{E}-03$} & \multicolumn{3}{|c|}{$9.3 E-05$} & \multicolumn{3}{|c|}{$1.8 \mathrm{E}-04$} \\
\hline \multicolumn{10}{|c|}{ Varying $T$} \\
\hline $\begin{array}{c}T \\
\left({ }^{\circ} \mathrm{C}\right) \\
\end{array}$ & \multicolumn{3}{|c|}{220} & \multicolumn{3}{|c|}{230} & \multicolumn{3}{|c|}{245} \\
\hline $\begin{array}{c}\lambda \\
(\mathrm{nm}) \\
\text { before printing }\end{array}$ & 1546.351 & 1546.473 & 1546.441 & 1546.3 & 1546.5 & 1546.5 & 1546.4 & 1546.4 & 1546.4 \\
\hline $\begin{array}{c}\lambda \\
(\mathrm{nm}) \\
\text { after printing } \\
\end{array}$ & 1535.215 & 1535.044 & 1545.594 & 1546.2 & 1546.2 & 1546.5 & 1537.0 & 1545.5 & 1536.1 \\
\hline $\begin{array}{c}\Delta \lambda \\
(\mathrm{nm})\end{array}$ & 11.136 & 11.429 & 0.847 & 0.122 & 0.29 & 0.021 & 9.378 & 0.902 & 10.276 \\
\hline$\Delta \lambda / \lambda=\Delta L / L$ & 0.00720147 & 0.007390365 & 0.000547709 & $8 \mathrm{E}-05$ & 0.0002 & $1 \mathrm{E}-05$ & 0.0061 & 0.0006 & 0.0066 \\
\hline $\begin{array}{l}\Delta \lambda_{a v} \\
(\mathrm{~nm})\end{array}$ & \multicolumn{3}{|c|}{$7.8 \mathrm{E}+00$} & \multicolumn{3}{|c|}{$1.4 \mathrm{E}-01$} & \multicolumn{3}{|c|}{$6.9 \mathrm{E}+00$} \\
\hline$(\Delta \lambda / \lambda)_{o v}$ & \multicolumn{3}{|c|}{$5.0 \mathrm{E}-03$} & \multicolumn{3}{|c|}{$9.3 \mathrm{E}-05$} & \multicolumn{3}{|c|}{$4.4 \mathrm{E}-03$} \\
\hline \multicolumn{10}{|c|}{ Varying cooling rate } \\
\hline $\begin{array}{l}\text { Cooling speed } \\
\text { (mm/min) }\end{array}$ & \multicolumn{3}{|c|}{600} & \multicolumn{3}{|c|}{1800} & \multicolumn{3}{|c|}{3600} \\
\hline $\begin{array}{c}\lambda \\
(\mathrm{nm}) \\
\text { before printing }\end{array}$ & 1546.321 & 1546.313 & 1546.235 & 1546.4 & 1546.3 & 1546.4 & 1546.4 & 1546.4 & 1546.4 \\
\hline $\begin{array}{c}\lambda \\
(\mathrm{nm}) \\
\text { after printing } \\
\end{array}$ & 1543.519 & 1533.778 & 1542.743 & 1537.1 & 1537.4 & 1534.8 & 1537 & 1545.5 & 1536.1 \\
\hline $\begin{array}{c}\Delta \lambda \\
(\mathrm{nm})\end{array}$ & 2.802 & 12.535 & 3.492 & 9.315 & 8,968 & 11.602 & 9.378 & 0.902 & 10.276 \\
\hline$\Delta \lambda / \lambda=\Delta L / L$ & 0.001812043 & 0.008106379 & 0.002258389 & 0.006 & 0.0058 & 0.0075 & 0.0061 & 0.0006 & 0.0066 \\
\hline $\begin{array}{l}\Delta \lambda_{a v} \\
(\mathrm{~nm})\end{array}$ & \multicolumn{3}{|c|}{$6.3 E+00$} & \multicolumn{3}{|c|}{$1.0 E+01$} & \multicolumn{3}{|c|}{$6.9 \mathrm{E}+00$} \\
\hline$(\Delta \lambda / \lambda)_{o v}$ & \multicolumn{3}{|c|}{$4.1 \mathrm{E}-03$} & \multicolumn{3}{|c|}{$6.4 \mathrm{E}-03$} & \multicolumn{3}{|c|}{ 4. $4 \mathrm{E}-03$} \\
\hline
\end{tabular}

Table 1. Summary of results obtained in packaging uniform gratings. Variations in package length, temperature and cooling rate are assessed without control of the applied load.

7. K. Cook, J. Canning, S. Leon-Saval, Z. Redi, Md. Hossain, J-E. Comatti, Y. Luo, G-D. Peng, Opt. Lett. 40 (17), 3966-3999, (2015).

8. P. E. Dyer, R. J. Farley, R. Giedl, K. C. Byron and D. Reid, Electron. Lett., 30, (11), 860-862, (1994).

9. J. Canning, M.G. Sceats, H.G. Inglis, P.Hill, Opt. Lett., 20, (21), 2189-2191, (1995).

10. J. Canning, Opt. Fibre. Tech, 6, 275-289, (2000).

11. J. Canning, (Inv), OSA Bragg Gratings, Photosensitivity and Poling (BGPP 2007), Quebec City, Canada, (2007).

12. J. Canning, Lasers \& Photon. Rev., 2(4), 275-289, Wiley, USA (2008).
13. http://www.matbase.com/material-categories/natural-and-syntheticpolymers/commodity-polymers/material-properties-of-acrylonitrilebutadiene-styrene-general-purpose-gp-abs.html

14. https://web.archive.org/web/20070504020850/http://machinistmaterials.com/comparison table for plastics.htm

15. P.C. Hill, B,J. Eggleton, Electronics Lett., 30 (14), 1172-1174, (1994).

16. https://www.abc.net.au/catalyst/fembots-robots/11007724

17. F. Ouellette, Opt. Lett. 12, 847-849 (1987).

18. C. S. Goh, M. R. Mokhtar, S. A. Butler, S. Y. Set, K. Kikuchi and M. Ibsen, IEEE Photon. Technol. Lett., 15 (4), 557-559, (2003). 


\section{Full citation listings}

[1] D. Vilarinho, A. Thoodoiou, C. Leitao and C. A. F. Marques, "POFPBembedded cork insole for plantar pressure monitoring", Sensors 7(12), 2924, (2017).

[2] Z. Hao, K. Cook and J. Canning, "Embedded smart FBG orthotics", $8^{\text {th }}$ Asia Pacific Opt. Sensors Conf. (APOS 2019), Auckland, New Zealand (2019).

[3] C. Martelli, J. Carlos Cardozo da Silva, D. Pipa, M. José da Silva, C.R. Zamarreño, J. Canning, P. E. Schaphauser, G. Dutra, R. Daciuk, J. R. Galvão, F. Mezzadri, A. B. Renzo, U. Dreyer, G. Weber, C. Janeczko and O. G. da Rocha, "Photonic sensors: from horse racing to horse power", SPIE Proc. V10323, 25th Int. Conf. on Optical Fiber Sensors, 103230W (2017). https://doi.org/10.1117/12.2272465

[4] G. W. Yoffe, Peter A. Krug, F. Ouellette, and D. A. Thorncraft, "Passive temperature-compensating package for optical fiber gratings," Appl. Opt. 34, 6859-6861 (1995).

[5] D.C. Psaila, H.G. Inglis, "Packaging of optical fibre Bragg gratings", IEEE Proc. 51 $51^{\text {st }}$ Electron. Components \& Tech. Conf. (2001).

[6] K. Cook, G. Balle, J. Canning, L. Chartier, T. Athanze, M.A. Hossain, C. Han, J-E. Comatti, Y. Luo and G-D. Peng, "Step-index optical fibre drawn from 3D printed preforms", Opt. Lett., 41 (19), 4554-4557, (2016).

[7] K. Cook, J. Canning, S. Leon-Saval, Z. Redi, Md. Hossain, J-E. Comatti, Y. Luo, G-D. Peng, "Air-structured optical fibre drawn from a 3Dprinted optical preform”, Opt. Lett. 40 (17), 3966-3999, (2015).
[8] P. E. Dyer, R. J. Farley, R. Giedl, K. C. Byron and D. Reid, "High reflectivity fibre gratings produced by incubated damage using a $193 \mathrm{~nm}$ ArF laser," Electron. Lett., 30, (11), 860-862, (1994).

[9] J. Canning, M.G. Sceats, H.G. Inglis, P.C. Hill., "Transient and permanent gratings in phosphosilicate optical fibres produced by the flash condensation technique", Opt. Lett., 20, (21), 2189-2191, (1995).

[10] J. Canning, "Photosensitisation and photostabilisation of laser-induced index changes in optical fibres", Opt. Fibre. Tech, 6, 275-289, (2000).

[11] J. Canning, "Gratings and grating devices in structured fibres using $193 \mathrm{~nm}$ from an ArF laser", (Inv), OSA Bragg Gratings, Photosensitivity and Poling (BGPP 2007), Quebec City, Canada, (2007).

[12] J. Canning, "Fibre Gratings \& Devices for Sensors \& Lasers", Lasers \& Photon. Rev., 2(4), 275-289, Wiley, USA (2008).

[13] http://www.matbase.com/material-categories/natural-and-syntheticpolymers/commodity-polymers/material-properties-of-acrylonitrilebutadiene-styrene-general-purpose-gp-abs.html

[14] https://web.archive.org/web/20070504020850/http://machinistmaterials.com/comparison table for plastics.htm

[15] P.C. Hill, B. J. Eggleton, "Strain gradient chirp of fibre gratings", Electronics Lett., 30 (14), 1172-1174, (1994).

[16] https://www.abc.net.au/catalyst/fembots-robots/11007724

[17] François Ouellette, "Dispersion cancellation using linearly chirped Bragg grating filters in optical waveguides," Opt. Lett. 12, 847-849 (1987)

[18] C. S. Goh, M. R. Mokhtar, S. A. Butler, S. Y. Set, K. Kikuchi and M. Ibsen, "Wavelength tuning of fiber Bragg gratings over $90 \mathrm{~nm}$ using a simple tuning package," in IEEE Photon. Technol. Lett., 15 (4), 557-559, 2003. 